\title{
Thyroid screening in pregnancy-no effect on child cognition?
}

$\mathrm{S}$ creening women for TSH and free $\mathrm{T}_{4}$ levels at the end of the first trimester of pregnancy and subsequent treatment of hypothyroidism does not have an observable effect on their children's IQ at 3 years of age, according to a novel study published in The New England Journal of Medicine.

Previous retrospective studies have shown an association between hypothyroidism in pregnancy and low IQ in children.

The new analysis, led by John Lazarus (Centre for Endocrine and Diabetes Sciences, Cardiff University School of Medicine) and Nicholas Wald (Wolfson Institute of Preventive Medicine, Barts and the London School of Medicine and Dentistry), was a randomized prospective study involving 21,846 pregnant women. All study participants underwent TSH and free $\mathrm{T}_{4}$ tests at $\sim 12$ weeks of gestation. Women assigned to the screening group received their test results immediately and underwent therapy to normalize serum thyroid hormone levels if the test results were considered positive, that is, TSH levels above the $97.5^{\text {th }}$ percentile and/or free $\mathrm{T}_{4}$ levels below the $2.5^{\text {th }}$ percentile. By contrast, serum test results for women in the

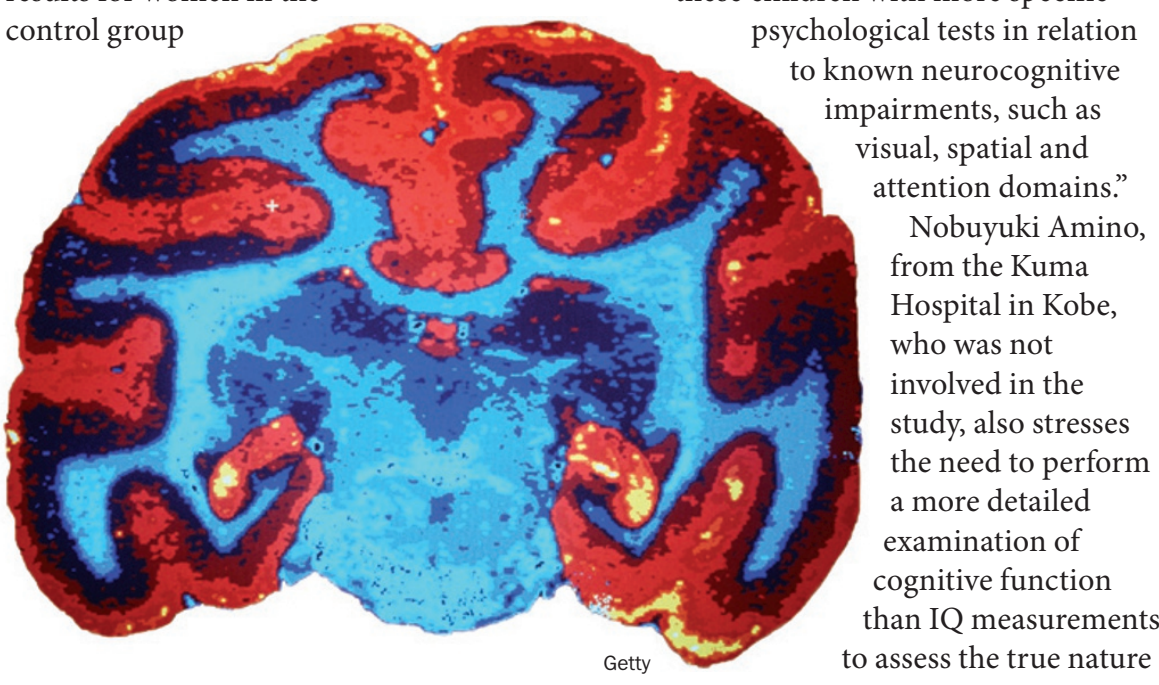

were not available until delivery and these women did not receive therapy for hypothyroidism. When the children of these mothers were aged 3 years, their IQ was measured by psychologists who were not aware of the maternal screening results.

The researchers were able to assess the IQ of $78.2 \%(n=390)$ of the children whose mothers had a positive test result in the screening group and $73.3 \%$ $(n=404)$ of the children whose mothers had a positive test in the control group. "We expected there to be a difference, given the findings of a previous study," comments Lazarus. However, and surprisingly, both the mean IQ scores and the proportion of children with an IQ score $<85$ were not significantly different between the two groups.

To explain the discrepancy between their findings and those of previous studies, the investigators highlight the randomized nature of their trial and suggest that earlier studies might have been subject to confounding. However, "more specific cognitive impairments that we did not assess may be associated with maternal hypothyroidism," states Lazarus. "We plan to assess these children with more specific chological tests in relation known neurocognitive pairments, such as visual, spatial and ention domains." mino, from the Kuma Hospital in Kobe, who was not involved in the study, also stresses the need to perform a more detailed examination of cognitive function to assess the true nature of the relationship between maternal mild hypothyroidism and child cognitive function. Amino also highlights that, in contrast to previous analyses, in the study by Lazarus and colleagues "the hypothyroid condition is very mild, which may be related to the absence of a significant difference between the groups".

According to Amino, limitations of the study include that the authors did not provide the normal IQ value of children born to mothers without thyroid problems and that they classified women as having a positive test result either when they had high TSH levels or low free $\mathrm{T}_{4}$ levels. "The exact nature of isolated low free $\mathrm{T}_{4}$ levels is not well understood and is a different condition from elevated TSH levels," explains Amino. Furthermore, the researchers adjusted the cut-off value of the TSH tests according to assay results obtained during the study. "At present, the TSH immunoassay is sensitive and reproducible enough to use the fixed value, and periodic change of the cutoff value might influence the consistency of the results," comments Amino. More prospective and randomized trials are needed to clarify this important issue.

The current guidelines for management of thyroid disease in pregnancy do not recommend routine screening for hypothyroidism. "Our findings suggest that screening for thyroid function at the end of the first trimester and treating mild hypothyroidism will make no difference to a child's IQ," says Lazarus. However, "the findings do not necessarily negate the strategy of screening, as there are other reasons to suggest that it may be beneficial," he adds.

\section{Joana Osório}

Original article Lazarus, J. H. et al. Antenatal thyroid screening and childhood cognitive function. N. Engl. J. Med. 366, 493-501 (2012) 